\title{
L’alpha de Cronbach, ses émules, la consistance interne, la fidélité : une mise au point
}

\author{
Louis Laurencelle ${ }^{\mathrm{a} \otimes}(0)$ \\ ${ }^{a}$ Université du Québec à Trois-Rivières
}

\begin{abstract}
Cronbach's alpha coefficient is one of the most widely used indices of the quality of a psychometric test, a popularity that has also earned it an abundance of criticism and sustained competition from a large number of candidates. Do these coefficients assess the "reliability" of the test or its "internal consistency", what do these designations mean, and how do the interpretations given to these indices relate to their mathematical structure? The historical and semantic drift of the term "reliability" and the obvious vagueness of the term "internal consistency" are the probable causes of this confusion. We examine these different questions in the light of Cronbach's 1951 article and try to set the record straight by advising the researcher to define a priori the quality of the test he is trying to verify and to ensure that the calculation procedure he chooses corresponds explicitly to this quality.
\end{abstract} Acting Editor $=$ Denis Cousineau (Université d’Ottawa)

Keywords $₫$ alpha de Cronbach, fidélité, consistance interne, dimensionnalité du test // Cronbach’s alpha, reliability, internal consistency, test dimensionality.

louis.laurencelle@gmail.com

10.20982/tqmp.17.1.p046

\section{Introduction}

La question de la valeur heuristique et de l'interprétation du coefficient alpha $(\alpha)$ de Cronbach revient périodiquement sur la table et, comme cette question répond à une préoccupation de plusieurs chercheurs en sciences humaines, il est pertinent de s'y pencher. Dans un article récent intitulé "L'alpha de Cronbach est l'un des pires estimateurs de la consistance interne : une étude de simulation ", les auteurs Bourque, Doucet, LeBlanc, DuPUis et NADEAU (2019) tentent de clarifier les notions afférentes à l'utilisation du coefficient $\alpha$ et, finalement, ils proposent de s'en passer. Notre but ici, en puisant dans la littérature classique sur le sujet, est de clarifier les notions psychométriques en jeu de même que la signification du coefficient $\alpha$ et de montrer que le $\alpha$ ainsi que ses compétiteurs plus récents (OsBuRN, 2000) racontent chacun sa propre histoire et qu'ils en révèlent peu sur la fidélité.

D’entrée de jeu, dans le dessein évident de définir le problème à l'étude, BouRQuE et collègues. (2019, p. 79) écrivent :
Les estimateurs de fidélité permettent d'estimer à quel point le score observé se rapproche du score vrai. Par exemple, les indices de consistance interne comme l'alpha de Cronbach sont des mesures de fidélité qui font référence au degré auquel les items mesurent le même concept (CRONBACH, 1951).

Or, précisément sur ce double énoncé, CRONBACH (1951, p. 298), dans son article original sur la question, dit :

L'approche classique du fractionnement $d u$ test ${ }^{1}$ en deux moitiés a été abondamment critiquée. L'une des critiques était que le bifractionnement ne fournit pas la même information que la corrélation prise entre deux tests équivalents administrés à des moments différents. Ce différend est purement d'ordre sémantique : les deux coefficients mesurent en fait des propriétés différentes et ne devraient pas être désignés par la même appellation de « fidélité ». Une nouvelle mesure faite après un intervalle et utilisant le même test identique indique jusqu'à quel point les scores

1. Cronbach ici réfère implicitement à toutes les formes du fractionnement du test composite, incluant celui en tous ses items constituants. 
sont stables, et l'on peut parler alors de coefficient de stabilité. La corrélation entre deux versions du test administrées pratiquement en même temps est un coefficient d'équivalence, montrant jusqu'à quel point deux mesures de la même caractéristique générale concordent. Un coefficient basé des versions comparables mais avec un intervalle entre les tests est un coefficient d'équivalence et de stabilité. [traduction libre]

Mutatis mutandis, il va sans dire que la démonstration de stabilité obtenue par la corrélation entre deux tests décalés dans le temps est aussi une démonstration d'équivalence, ce qui n'est pas le cas si on inverse les rôles des deux approches.

La propriété de consistance interne d'un test n'a pas encore reçu de définition claire et communément acceptée dans la littérature. D’aucuns parlent plutôt de cohérence interne, d'autres d'homogénéité, certains référant plus explicitement à la dimensionnalité du test, voire à la présence d'un seul facteur. Tous se réfèrent à la quantité de liens que les parties d'un test composite entretiennent entre elles. Comparativement, c'est sommairement qu'on parlera de la fidélité d'un test, tels un test de QI ou aussi bien un test de force maximale du bras ou une mesure du poids corporel. Ce dont le coefficient de fidélité classique, noté $r_{X X}$, nous informe est entendu comme une caractéristique distinctive de la personne mesurée, sa valeur étant stable dans le temps, comparable d'un instrument à l'autre et permettant de classer la personne selon cette valeur par comparaison avec d'autres individus de valeurs connues. Que le poids corporel reflète l'âge de la personne, son état de santé, son développement musculaire, son obésité, voilà des questions d'interprétation contextuelle, qui n’ont pas partie liée avec la fidélité du score lui-même et qui nécessitent une autre analyse, plus contextuelle et individualisée. Le QI lui-même, l'un des produits les plus glorieux de la psychométrie, doté d'une fidélité excellente, ${ }^{2}$ a connu plusieurs ordres d'interprétation : niveau global des habiletés mentales de l'individu, indice de 'scolaptitude', niveau de développement cognitif, etc., la question étant encore en débat. Il reste que le QI est composite, comme plusieurs autres tests psychométriques, et sa valeur résultante est basée sur plusieurs échelles et sur d'encore plus nombreux items : il ne peut pas être ici question d'équivalence globale des contenus, d'homogénéité de contenu ni d'unidimensionnalité. ${ }^{3}$ Ainsi, pour un QI issu d'un test comportant 100 items et à fidélité $r_{X X}$ typique de 0,90 , le score produit est réputé globalement fiable, caractéristique de la personne mesurée (avec une marge d'erreur définie) et stable dans le temps : est-il homogène ou " consistant " ou à contenu factoriel simple? En fait, les nombreuses échelles qui le constituent sont chacune autant de modalités, d'habiletés, de caractéristiques, de facettes différentes contribuant au résultat global. De plus, au niveau de la consistance statistique du test telle qu'évaluée par l'intercorrélation entre les items, une formule du même Cronbach (1951; voir aussi le concept d' ' unitarisation » dans Laurencelle, 1998, pp. 82 et 122) nous permet d'estimer approximativement ce que serait la corrélation moyenne entre les items, soit :

$$
\overline{r_{i, j}}=\frac{\alpha}{n+(1-n) \alpha}
$$

(CRONBACH, 1951, éq. 44), où $n$ est le nombre d'item. Cette formule s'applique à tout indice global " de consistance " d'un test composite, incluant la fidélité $r_{X X}$. Pour un test comportant $n=100$ items et à fidélité 0,90 , le lecteur vérifiera que la corrélation moyenne inter-item est d'environ 0,083. Le nombre d'items trouvés dans les tests de QI avoisine plutôt les 200, auxquels cas nous obtenons $\overline{r_{i, j}} \approx 0,043$. Enfin, CRONBACH (1951, p. 300) explique qu'on peut obtenir une bonne corrélation entre deux ensembles d'items même si les items de chaque ensemble ont des intercorrélations nulles, à condition que chaque item d'un ensemble retrouve son jumeau dans l'autre ensemble. Guttman en 1954, dans sa célèbre " théorie des facettes ", va plus loin en proposant que les facettes ou composants d'un concept psychométrique sont essentiellement indépendantes (il admettra plus tard la possibilité de liens) et que le concept qu'elles expriment est créé par leur assemblage. ${ }^{4}$ Dans un tel cas, pas de consistance ni de cohérence internes statistiquement démontrables, et cela pour un objet psychométrique qui peut néanmoins avoir une existence et une fidélité. CRONBACH (1951) note enfin que, si le contenu conceptuel simple du test analysé facilite l'interprétation des scores produits, un contenu nuancé, reposant sur des sous-ensembles d'items, des facettes plus ou moins intercorrélées, sera lui aussi interprétable.

Si l'homogénéité, l'unidimensionnalité ou la cohérence interne d'un test reflètent le niveau d'intercorrélation des composants ou items du test, la consistance interne quant à elle pourrait référer à la masse totale des valeurs vraies représentées par les parties du test, que ces parties soient intercorrélées ou non, un point de vue qui réunit à la fois la théorie des facettes de GuTTMAN (1954) et l'analyse de Cronbach, et qui trouve, inopinément, son expression dans

2. Le coefficient de fidélité $r_{X X}$ des tests de QI modernes jouxte ou déborde la valeur 0,90.

3. Le modèle factoriel d'un test de QI comme d'autres tests d'aptitude peut néanmoins contenir un facteur à variance (très dominante), pour laquelle on parlerait alors d'« unidimensionnalité essentielle » (STOUT, 1987), ou encore un facteur surordonné, couvrant l'intercorrélation de facteurs de premier ordre, ces deux structures étant évidemment multifactorielles.

4. Dans un tel cas, nul item ne contiendra une partie de la « valeur vraie » du concept, seule leur conjonction définissant cette valeur. 
le modèle factoriel complet de la théorie des tests (LAURENCELLE, 1998).

\section{Le modèle factoriel de la théorie des tests et ses impli- cations}

Le modèle de base. Dans leur article, Bourque et collègues. (2019) rappellent succinctement le modèle de base de la théorie des tests, lequel modèle est aussi celui de toute mesure instrumentale : l'opération de mesure fournit une donnée qui représente par un nombre la grandeur de l'objet ou de la caractéristique évaluée Ce nombre peut être altéré par une erreur d'approximation, soit :

$$
\begin{aligned}
X & =f(\text { grandeur de l'objet })+\text { erreur d'approximation } \\
& =V+e
\end{aligned}
$$

où ' $V$ ', dite " valeur vraie ", dénote la grandeur réelle et réputée fixe de l'objet évalué, et ' $e$ ', dite 'erreur de mesure' ou 'erreur', toute variation numérique à caractère aléatoire et d'espérance zéro autour de la valeur vraie. L'erreur est réputée indépendante de la valeur vraie. Dans le contexte de ce modèle simple, la variance des mesures $X$ est donc:

$$
\sigma^{2}(X)=\sigma^{2}(V)+\sigma^{2}(e) .
$$

La variance vraie pouvant être considérée de l'information sur les grandeurs mesurées et la variance d'erreur du bruit, le quotient de la variance vraie sur la variance totale observée représente la charge d'information transmise par un système de mesure et est traditionnellement dénommée fidélité (reliability, en anglais) soit :

$$
r_{X X}=\sigma^{2}(V) / \sigma^{2}(X) .
$$

L'estimateur naturel pour le coefficient $r_{X X}{ }^{5}$ est la corrélation entre deux mesures des mêmes objets, $X_{1}$ et $X_{2}$, mieux notés par $V+e_{1}$ et $V+e_{2}$. Leur corrélation est alors (où 'cov' dénote la covariance) :

$$
\begin{aligned}
r_{X X}= & \operatorname{cov}\left(X_{1}, X_{2}\right) /\left(\sigma_{X} \sigma_{X}\right) \\
= & {\left[\operatorname{cov}\left(V+e_{1}, V+e_{2}\right)\right] / \sigma_{X}^{2} } \\
= & {\left[\operatorname{cov}(V, V)+\operatorname{cov}\left(V, e_{2}\right)+\operatorname{cov}\left(e_{1}, V\right)+\right.} \\
& \left.\quad \operatorname{cov}\left(e_{1}, e_{2}\right)\right] / \sigma_{X}^{2} \\
= & \sigma_{V}^{2} / \sigma_{X}^{2},
\end{aligned}
$$

(toutes covariances avec les termes $e$ étant nulles). Quant à la corrélation entre la mesure et la valeur vraie, nous avons :

$$
\begin{aligned}
r_{X V} & =\operatorname{cov}(X, V) /\left(\sigma_{X} \times \sigma_{V}\right) \\
& =\operatorname{cov}(V+e, V) /\left(\sigma_{X} \times \sigma_{V}\right) \\
& =[\operatorname{cov}(V, V)+\operatorname{cov}(e, V)] /\left(\sigma_{X} \times \sigma_{V}\right) \\
& =\sigma_{V}^{2} /\left(\sigma_{X} \times \sigma_{V}\right) \\
& =\sigma_{V} / \sigma_{X}=\sqrt{r_{X X}},
\end{aligned}
$$

ces résultats classiques clarifiant une ambiguïté de désignation et de calcul dans BouRQuE et collègues. (2019, p. 81).

Le modèle factoriel. La valeur vraie $(V)$ caractérise, avec plus ou moins de précision, la grandeur de l'objet visée par l'opération de mesure, cette grandeur pouvant ressortir à différentes sources ou facettes. C'est le cas pour le poids corporel, qui est une résultante de plusieurs facteurs, et ce l'est encore plus pour la plupart des 'grandeurs' auxquelles s'intéresse la psychométrie, tels l'estime de soi, l'intérêt pour les sciences, l'habileté mentale. La valeur vraie peut donc être conçue comme un amalgame, un total d'effets quantitatifs ressortissant chacun à une source, un facteur particulier. C'est ce qu'est destiné à représenter le modèle factoriel de la théorie des tests, principalement basé sur l'analyse factorielle (par axes principaux). Selon ce modèle, la variance du score $X$ comporte :

1. une part d'erreur aléatoire (et donc non corrélée), plafonnant la fidélité du score;

2. une part de variance partagée par une ou plusieurs autres mesures $(Y, Z$, etc.), cette 'variance commune' étant modélisée par un ou plusieurs axes mathématiques dénommés facteurs;

3. une part de variance propre, invisible par l'analyse factorielle, soit, au total: $\sigma_{X}^{2}=c_{X_{1}} \cdot \sigma^{2}\left(f_{1}\right)+c_{X_{2}} \cdot \sigma^{2}\left(f_{2}\right)+$ $\cdots+\sigma^{2}$ (propre) $+\sigma_{e}^{2}$.

La variance commune et la variance propre constituent la variance vraie. L'analyse factorielle classique représente essentiellement la variance commune, aussi appelée 'communauté', soit la somme des variances transférées sur le ou les facteurs retenus. Cette analyse ignore essentiellement la variance propre, cette dernière pouvant être estimée par la différence :

$$
\sigma^{2}(\text { propre })=r_{X X}-\text { communauté } \geq 0
$$

Cette 'variance propre', associée à nulle autre variable du test que celle considérée, fait écho à l'idée de facettes potentiellement indépendantes qui participeraient à leur façon à la formation du concept psychométrique ciblé par le test et matérialisé par le score $X$. Un tel test, comme argumenté ci-dessus, pourrait présenter une

5. Le symbole utilisé pour désigner la fidélité varie d'un auteur à l'autre, utilisant parfois le $r$ désignant le coefficient de corrélation de Pearson empirique, parfois son pendant paramétrique $\rho$, et ce habituellement sans exposant (p. ex. $\rho\left(X_{j}, X_{j}^{\prime}\right)$ dans Thorndike, 1950, p. 374) ou rarement avec exposant 2 (p. ex. $\rho_{X}^{2}$ dans TRAUB (1994), la première forme étant isomorphe à la formule d'estimation, la seconde, à l'ordre des éléments de variance résultants. La forme $r_{X X}$ semble toutefois prédominante. 
fidélité $r_{X X}$ valable telle qu'établie par la procédure classique tout en ne manifestant aucune cohérence ou homogénéité appréciable via le coefficient $\alpha$ ou ses émules, voire aucune solution factorielle.

Comme le recommande instamment CRONBACH (1951, p. 298, voir plus haut), il faut donc distinguer clairement fidélité (reliability en anglais ${ }^{6}$ ) et consistance interne ou cohérence (internal consistency). Le premier révèle que le score obtenu est caractéristique de l'objet mesuré, peut être reproduit (plus ou moins sans erreur) à tout moment et peut servir fiablement à le classer parmi d'autres, le second concept indiquant que la mesure obtenue émane de l'assemblage de caractéristiques de l'objet plus ou moins redondantes, leur convergence globale, si elle existe, permettant d'en circonscrire l'interprétation. Deux indices donc, complémentaires, l'un n'impliquant pas l'autre.

\section{Que retirer de cette mise au point}

L'histoire de la statistique appelée " alpha de Cronbach " remonte à 1937, avec l'article publié par Kuder et Richardson dans le $2^{e}$ numéro de la célèbre revue américaine Psychometrika, le calcul étant alors appliqué à un test à items binaires. Le coefficient a réapparu sporadiquement, sous une forme ou une autre, incluant celle qu'a consacrée Cronbach, jusqu'à son article de 1951, où il écrit (p. 299) :

Nous proposons le symbole $\alpha$ en partie pour sa simplicité. "Formule 20 de KuderRichardson " est une dénomination malcommode pour un outil auquel nous prédisons une importance grandissante dans la littérature psychométrique.

Les citations déjà faites de cet article ainsi que les autres analyses et remarques qu'on y trouve montrent que l'auteur avait vu dans ce coefficient la plupart des significations et limitations qu'on semble redécouvrir aujourd'hui, et qu'il anticipait déjà ses réflexions tardives (et publiées post mortem en 2004) dans lesquelles il déplore que les utilisateurs qui citent et utilisent (ou critiquent) le coefficient $\alpha$ n'ont, pour la plupart, pas lu l'article de 1951, reconnaissant en même temps que le $\alpha$ n'exprime pas tout ce qu'il y a connaître dans un test. En page 413, CRONBACH (2004) conclut même :

Je suis persuadé que l'erreur-type de mesure [obtenue généralement par l'équation $\left.\sigma_{\mathrm{X}} \sqrt{1-r_{\mathrm{XX}}}\right]$... est la plus importante information à donner sur un instrument, plutôt qu'un coefficient.
Pour conclure cette mise au point, voici quelques réflexions que nous invitons le lecteur à méditer :

1. Quelle sorte de fidélité le chercheur veut-il faire valoir? Pour paraphraser CRONBACH (1951), s'il veut caractériser la fiabilité du score (total) du test, sa stabilité dans le temps et sa précision, c'est au 'coefficient de stabilité' $\left(r_{X X}\right)$, le coefficient de fidélité classique, qu'il doit recourir. S’il cible plutôt la recette de composition du score total et les interrelations en ses éléments, ce serait alors à un 'coefficient d'équivalence', mieux nommé consistance interne, qu'il devrait s'adresser, un indice bien représenté par le $\alpha$ de Cronbach et dont d'autres versions à interprétations différentes sont disponibles (voir p. ex. OsBURN, 2000).

2. Le meilleur indice qui caractérise la précision d'un score ou d'une mesure reste l'erreur-type de mesure, qui indique simplement et dans les unités naturelles de la mesure la marge d'erreur attendue qu'elle comprend. Sur un pèse-personne gradué en dixièmes de kilogrammes, on rapportera par exemple $52,2 \pm 0,1$ $\mathrm{kg}$. Pour un test psychométrique typique, on indiquera par exemple un QI par $112 \pm 5$ unités, en supposant une moyenne de 100, un écart-type de 15 et une fidélité $r_{X X}$ de 0,90. Rappelons que l'indice $r_{X X}$ lui-même comme le coefficient $\alpha$ et tous ses émules sont exprimés en unités pures, standardisés, de sorte qu'un $\alpha$ de 0,95 peut correspondre à une erreur-type inacceptable parce que trop grande dans un contexte donné alors que, dans d'autres cas, une basse valeur d' $\alpha$ (ou de $\omega, \lambda$, etc.) pourra correspondre à une marge d'erreur convenable.

3. Le coefficient $\alpha$ a d'entrée de jeu été montré comme égal à la moyenne des corrélations possibles entre les scores de toutes les bipartitions possibles d'un test de $n$ items. Rappelons que son calcul, comme celui de la plupart de ses émules, est basé sur les valeurs brutes des items sans standardisation. Or, il est facilement démontré que, pour des items à intercorrélations égales, l'inégalité de leurs variances correspondra à un coefficient $\alpha$ plus bas que si les variances appliquées étaient égales, les calculs de base du coefficient étant effectués sur des covariances. À ce titre, il est faux d'affirmer que sous cette condition le coefficient $\alpha$ sousestime la fidélité du score $X$, le score $X$ lui-même étant soumis à l'effet de ces inégalités. Pour un ensemble d'items à variances toutes égales, l' $\alpha$ est exactement calculable (par la formule dite de Spearman-Brown) à partir de l'intercorrélation moyenne entre items, sinon les items à variance plus forte auront plus d'influence

6. Reliable signifie fiable, sur quoi l'on peut compter, sa traduction française " fidèle " étant passée à l'histoire. Quant au terme anglais fidelity, il n’apparaît que rarement dans la littérature psychométrique anglo-saxonne et sa signification reste flottante, correspondant parfois à notre " fidélité " mais aussi à " conformité " (dans le sens de copie fidèle), la corrélation d'une version du test avec une autre, ou une plus courte ou plus longue, exprimant cette conformité. 
à la fois sur le score $X$ et sa variance et sur le coefficient $\alpha$, ce qui est à nos yeux un mérite d'exactitude à préserver.

4. Mathématiquement parlant, le coefficient $\alpha$ est l'expression standardisée de la covariance moyenne entre les items d'un ensemble, et sa valeur possible va de $-\infty$ à 1 . Ladite covariance moyenne peut ressortir à un seul facteur commun (unidimensionnel) ou à deux ou plusieurs facteurs, comme le reconnaît CRONBACH (1951). Prenons l'exemple d'un test à 6 items formé de deux groupes de 3 items. Supposant des variances d'items égales, des corrélations de 0,9 dans chaque groupe et de 0,0 entre les groupes : l' $\alpha$ résultant sera de 0,771 . Posons maintenant une corrélation de 0,5 entre les items des deux groupes, ce qui est une situation vraisemblable : l' $\alpha$ monte alors à 0,921 . Or, si le test est « consistant " parce qu'il a une bonne dose d'interdépendance entre ses items, il n'est pas " homogène " mais plutôt bi-factoriel, ce qui n'est pas un défaut en soi mais rend seulement son interprétation plus nuancée, comme le remarque encore CRONBACH (1951).

5. Le test est-il homogène, y a-t-il de la corrélation entre ses composants d'erreur, peut-on bien caractériser ce qu'il mesure, quelle confiance peut-on donner à l'interprétation de ses scores? À toutes ces questions, seule une étude réfléchie et ciblée peut espérer répondre, et l'analyse factorielle exploratoire par facteurs principaux (AFE), à l'avis de plusieurs spécialistes, reste l'outil incontournable . On y a déjà recours pour le calcul de certains indices compétiteurs du coefficient de Cronbach. L'AFE a été créée dans ce but, l'atteinte de ce but supposant un investissement qui déborde largement le calcul de n'importe quel indice ponctuel.

Aucune de ces questions cependant, ni de ces calculs, n'informe directement que le test est fidèle (c.-à-d. à mesure reproductible) et précis (c.-à-d. fournissant des scores à marge d'erreur déterminée), ce qui est une autre histoire.

\section{Références}

Bourque, J., Doucet, D., LeBlanc, J., Dupuis, J. \& NAdeau, J. (2019). L'alpha de Cronbach est l'un des pires estimateurs de la consistance interne : une étude de simulation. Revue des sciences de l'éducation, 45(2), 78-99.

CRONBACH, L. J. (1951). Coefficient alpha and the internal structure of tests. Psychometrika, 16, 297-334.

CRONBACH, L. J. (2004). My current thoughts on coefficient alpha and successor procedures. Educational and psychological measurements, 64, 391-418.

GutTman, L. (1954). Some necessary conditions for common-factor analysis. Psychometrika, 19(2), 149161.

KUDER, G. F. \& RichARDSON, M. W. (1937). The theory of the estimation of test reliability. Psychometrika, 2, 151160.

LaURenCelle, L. (1998). Théorie et techniques de la mesure instrumentale. Québec : Presses de l'Université du Québec.

OsBuRn, H. G. (2000). Coefficient alpha and related internal consistency reliability coefficients. Psychological Methods, 5, 343-355.

STоUт, W. F. (1987). A nonparametric approach for assessing latent trait unidimensionality. Psychometrika, 52, 589-617.

THORNDIKE, R. L. (1950). Reliability, dans E. F. Lindquist (dir.), Educational measurement (, 560-620.

Traub, R. E. (1994). Reliability for the social sciences. Theory and applications (vol. 3.

\section{Citation}

LAURENCELle, L. (2021). L’alpha de Cronbach, ses émules, la consistance interne, la fidélité : une mise au point. The Quantitative Methods for Psychology, 17(1), 46-50. doi :10.20982/tqmp.17.1.p046

Copyright (c) 2021, LAURENCELLE. This is an open-access article distributed under the terms of the Creative Commons Attribution License (CC BY). The use, distribution or reproduction in other forums is permitted, provided the original author(s) or licensor are credited and that the original publication in this journal is cited, in accordance with accepted academic practice. No use, distribution or reproduction is permitted which does not comply with these terms.

Received: 20/11/2020 Accepted: 24/12/2020 